\title{
Chest computed tomography features of heart failure: A prospective observational study in patients with acute dyspnea
}

Kristina Miger $^{1} \oplus$, Andreas Fabricius-Bjerre ${ }^{1}$, Anne Sophie Overgaard Olesen ${ }^{1}$,

Ahmad Sajadieh ${ }^{1}$, Nis Høst ${ }^{1}$, Nanna Køber ${ }^{1}$, Annemette Abild ${ }^{2}$, Mathilde Marie Winkler Wille ${ }^{3}$, Jesper Wamberg ${ }^{4}$, Lars Pedersen ${ }^{5}$, Hans Henrik Lawaetz Schultz ${ }^{5}$, Christian Torp-Pedersen ${ }^{6,7}$, Olav Wendelboe Nielsen ${ }^{1,8,9}$

${ }^{1}$ Department of Cardiology, Bispebjerg and Frederiksberg Hospital, Denmark

${ }^{2}$ Department of Radiology, Bispebjerg and Frederiksberg Hospital, Denmark

${ }^{3}$ Department of Radiology, Nordsjaellands Hospital, Denmark

${ }^{4}$ Department of Emergency Medicine, Bispebjerg and Frederiksberg Hospital, Denmark

${ }^{5}$ Department of Pulmonary Medicine, Bispebjerg and Frederiksberg Hospital, Denmark

${ }^{6}$ Department of Cardiology, Nordsjaellands Hospital, Denmark

${ }^{7}$ Department of Cardiology, Aalborg University Hospital, Aalborg, Denmark

${ }^{8}$ Copenhagen Center for Translational Research Copenhagen University Hospital,

Bispebjerg and Frederiksberg Hospital, Denmark

${ }^{9}$ Faculty of Health and Medical Sciences, Copenhagen University, Denmark

\begin{abstract}
Background: Pulmonary congestion is a key component of heart failure (HF) that chest computed tomography (CT) can detect. However, no guideline describes which of many anticipated CT signs are most associated with HF in patients with undifferentiated dyspnea.

Methods: In a prospective observational single-center study, we included consecutive patients $\geq 50$ years admitted with acute dyspnea to the emergency department. Patients underwent immediate clinical examination, blood sampling, echocardiography, and CT. Two radiologists independently evaluated all images. Acute HF (AHF) was adjudicated by an expert panel blinded to radiology images. LASSO and logistic regression identified the independent $C T$ signs of $A H F$.

Results: Among 232 patients, 102 (44\%) had AHF. Of 18 examined CT signs, 5 were associated with AHF (multivariate odds ratio, 95\% confidence interval): enlarged heart (20.38, 6.86-76.16), bilateral interlobular thickening (11.67, 1.78-230.99), bilateral pleural effusion (6.39, 1.98-22.85), and increased vascular diameter (4.49, 1.08-33.92). Bilateral ground-glass opacification $(2.07,0.95-4.52)$ was a consistent fifth essential sign, although it was only significant in univariate analysis. Eighty-eight (38\%) patients had none of the five CT signs corresponding to a 68\% specificity and 86\% sensitivity for AHF, while two or more of the five CT signs occurred in 68 (29\%) patients, corresponding to $97 \%$ specificity and $67 \%$ sensitivity. A weighted score based on these five CT signs had an 0.88 area under the curve to detect $A H F$. Conclusions: Five CT signs seem sufficient to assess the risk of AHF in the acute setting. The absence of these signs indicates a low probability, one sign makes AHF highly probable, and two or more CT signs mean almost certain AHF. (Cardiol J 2022; 29, 2: 235-244)
\end{abstract}

Key words: pulmonary congestion, acute heart failure, chest computed tomography, dyspnea, emergency department

Address for correspondence: Kristina Cecilia Miger, MD, Bispebjerg University Hospital, Ebba Lunds Vej 40A, 2400 Copenhagen NV, Denmark, tel: +4560530520, e-mail: kristina.cecilia.miger@regionh.dk

Received: 6.07.2021

Accepted: 5.12.2021

Early publication date: 25.01.2022

This article is available in open access under Creative Common Attribution-Non-Commercial-No Derivatives 4.0 International (CC BY-NC-ND 4.0) license, allowing to download articles and share them with others as long as they credit the authors and the publisher, but without permission to change them in any way or use them commercially. 


\section{Introduction}

Heart failure (HF) is a complex syndrome with structural and/or functional cardiac abnormality corroborated by objective evidence of cardiogenic pulmonary or systemic congestion [1]. While the primary diagnosis is based on clinical findings, echocardiography, and natriuretic peptides [2], the diagnosis can be especially challenging in patients with comorbidities, which in many cases complicate the clinical picture. In many of such cases congestion signs are associated with uncertainty, especially the clinical signs and chest X-ray (CXR), which may not rule out acute HF (AHF) [2-4]. Furthermore, natriuretic peptides can also falsely be elevated in patients with acute comorbid conditions [3]. Lung ultrasound may support a diagnosis of $\mathrm{HF}$ in severe cases but is less useful for detecting mild degrees of congestion $[2,5,6]$.

Chest computed tomography (CT) is the gold standard for diagnosing interstitial lung disease, but it is not a recommended test for AHF [2, 7]. However, acute patients with dyspnea often undergo $\mathrm{CT}$ as the primary or secondary examination in the search for pulmonary embolus, acute coronary syndrome, or suspected pulmonary infection [8]. Therefore, CT has great potential to supplement or improve the diagnostic work up of patients with dyspnea, including AHF, either with a high negative predictive value or as a confirmatory test.

Because the utilization of CT in emergency departments (ED) has increased significantly in patients admitted with dyspnea [9], it could be an asset in the early detection of HF. Furthermore, due to several advances in cardiac $\mathrm{CT}$, it is perceivable that future iterations of $\mathrm{HF}$ guidelines will include an expanded role for cardiac CT [10]. First, however, it is essential to identify the strengths and limitations of using this modality for diagnosing congestion as a sign of HF. Although the literature describes several radiological features of pulmonary congestion in patients with known cardiac disease [11-15], we could not find a systematic approach to utilize CT for estimating the likelihood of AHF in consecutive dyspneic patients $[16,17]$. Because no standard methods exist to evaluate the additional value of the $\mathrm{CT}$, we have conducted a study to examine which CT features are most important to estimate the probability of AHF. To avoid selection bias, we performed an upfront extra chest CT in every consecutive patient.

The study aimed to identify which signs from $\mathrm{CT}$ are most associated with AHF in consecutive breathless patients and how to balance their im- portance. Secondly, we examined how these signs would perform in the diagnosis of AHF by internal validation.

The implication is that greater awareness is needed of the utilization of CT as a supplement to current guideline-recommended diagnostic tests for early detection and/or exclusion of HF.

\section{Methods}

\section{Design}

A prospective observational study in consecutive patients with acute dyspnea admitted to the ED at Bispebjerg University Hospital, Copenhagen, Denmark. The National Ethics Committee approved the study on Health Research Ethics, Denmark (Project-id: H-17000869).

\section{Population}

We screened consecutive patients $\geq 50$ years old in the ED on weekdays between 08:00 and 14:30 on 216 randomly selected days from November 2017 to August 2019. The main inclusion criterion was self-reported dyspnea, supported by at least one abnormal respiratory parameter (Fig. 1). The age limit of 50 years was chosen due to an increased risk of radiation in subjects below 50 years old $[18,19]$, and because HF is a more frequent differential diagnosis in patients aged $>50$ years.

The main exclusion criteria were a need for inotropic treatment, intensive care, and mechanical or non-mechanical ventilation. Patients were excluded if the CT or echocardiography could not be performed within 12 hours, or if patients were unwilling or unable to give consent (e.g., dementia) (Fig. 1).

All patients underwent immediate phlebotomy, clinical examination, comprehensive echocardiography, and a non-contrast CT.

\section{Clinical evaluation}

The first attending ED physician performed a systematic collection of the medical history, vital signs, and physical examination.

\section{Echocardiography}

Performed by experienced cardiologists according to the 2016 European Society of Cardiology guidelines [3], including systematic evaluation of diastolic function and left ventricular (LV) filling pressure [20].

The protocol emphasized LV ejection fraction (LVEF), E/A, E/ e', left atrium index volume, 


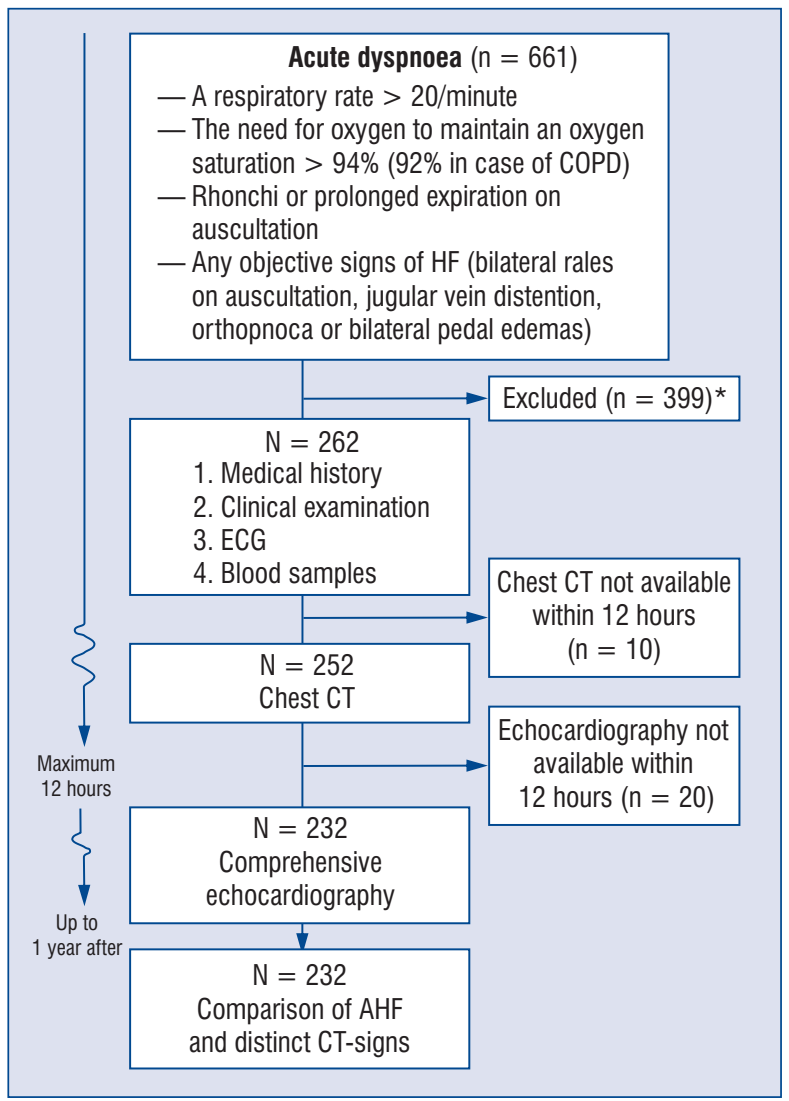

Figure 1. Flowchart of the inclusion process. The inclusion criteria were dyspnea and at least one objective sign of dyspnea. Figure 1 illustrates a flow chart over the inclusion and exclusion process; AHF — acute heart failure; CT - computed tomography; ECG - electrocardiogram; HF — heart failure; COPD - chronic obstructive pulmonary disease. *Excluded: not able to give consent (i.e., dementia, language barrier; $n=155$ ), unwilling to give written consent, not Danish citizen; $\mathrm{n}=117$ ), CT thorax already indicated (pulmonary embolism, aorta dissection or aneurysm; $\mathrm{n}=28$ ), acute coronary syndrome $(n=58)$, circulatory instability (need for inotropics; $n=3$ ), respiratory instability (mechanical/ /non-mechanical ventilation; $\mathrm{n}=19$ ), suspected life expectancy $<3$ months $(\mathrm{n}=19)$.

and quantification of severe valvular disease. Right ventricular parameters were free wall s', e', tricuspid annular plane systolic excursion, and tricuspid regurgitation gradient (TR). LVEF was evaluated by the Simpson method and wall motion score index, supported by other measures such as fractional shortening and mitral annular plane systolic excursion depending on image quality. The examinations were performed using an EPIQ 5 - Ultrasound system (Philips), X5-1 transducer (Philips) and subsequently evaluated on an Intelli-
Space Cardiovascular 4.1.0.0 (Philips). Recording and readings were approved by expert cardiologists accredited in echocardiography. Cardiac dysfunction was hierarchically classified as severe valve disease (HFVHD), reduced LVEF $<40 \%$ (HFrEF), mildly reduced LVEF from $40 \%$ to $49 \%$ (HFmrEF), and preserved LVEF (HFpEF) [3, 20]. Presence of $\mathrm{LV}$ diastolic dysfunction in this study required at least three of the four following measures: lateral e' $<10 \mathrm{~cm} / \mathrm{s}$, average E/e' ratio $>14$, left atrium maximum volume index $>34 \mathrm{~mL} / \mathrm{m}^{2}$, and peak TR velocity $>2.8 \mathrm{~m} / \mathrm{s}$ [20]. Elevated LV filling pressure was classified according to a previous consensus paper [20]. The interpreting cardiologists were always blinded to the CT findings, and in the case of disagreement, a third cardiologist evaluated the echocardiogram and case history.

\section{Definition of AHF diagnosis}

Two cardiologists adjudicated AHF by reviewing echocardiography, clinical data, blood samples, and medication, and disagreements were discussed with a third cardiologist. We assessed all components necessary to diagnose AHF [1] with a special emphasis on the echocardiographic signs of congestion as inferred from an elevated LV filling pressure in combination with cardiac dysfunction or an acute trigger for HF. Notably, the cardiologists did not evaluate radiology CXR or CT images to avoid circular reasoning. Instead, confirmation of pulmonary congestion was primarily supported by elevated LV filling pressure (grade II or grade III) according to recommendations $[3,21]$. If the LV filling pressure was indeterminate, other clinical information was considered supportive of the diagnosis, such as loop diuretic in the presence of evidence of systemic congestion, elevated natriuretic peptides, or the presence of a relevant trigger for AHF (conditions that improved significantly after treatment, such as tachyarrhythmias or markedly elevated blood pressure [3]).

\section{Definition of technical AHF diagnosis}

We established a technical AHF diagnosis, unbiased by radiology, based only on medical record registered data. This technical AHF diagnosis required the presence of all four objective criteria: I) HFrEF, HFmrEF, HFpEF, or HFVHD; II) N-terminal pro-B-type natriuretic peptide (NT-proBNP) > $300 \mathrm{pg} / \mathrm{mL}$; III) echocardiographic signs of elevated LV pressure, orthopnea, bilateral rales on auscultations or administrated intravenous loop diuretics, and IV) no acute pulmonary disease. 


\section{Radiology}

The CT was performed without contrast, with a radiation dose $<2 \mathrm{mSv}$ using a multislice CT scanner (Somatom Definitions Flash, Siemens Medical Solutions, Forschheim, Germany). The CT scans were obtained with the scan parameters: $140 \mathrm{kV}, 35 \mathrm{mAs}$ for each X-ray tube, 0.5 -ms gantry rotation, $128 \times 0.6 \mathrm{~mm}$ collimation with pitch of 1.2 , reconstructed slice thickness $3 \mathrm{~mm}$, using two different convolution kernels: a window of lung fields (I70f very sharp) with coronal and sagittal reformatted images and a soft tissue window (I40f).

\section{Interpretation of pulmonary congestion on CT}

Two radiologists, specialized in thoracic imaging and working at two different university hospitals, evaluated all CT scans. The radiologists were blinded to each other, to previous radiological reports, and to all clinical data. A pre-defined protocol dictated which CT signs to evaluate based on the Fleischner Society list of potential patterns: ground glass opacities, interlobular thickening, interlobar effusion, consolidation, crazy paving, and atelectasis, modified by adding peribronchial cuffing, enlarged heart, and increased vascular diameter [22, 23]. A CT sign was defined as positive if both radiologists reported its presence.

\section{Statistics}

All statistical analyses were performed using $\mathrm{R}$ Version 3.6.2 [24]. Continuous variables are presented with the mean and median, $95 \%$ confidence interval (CI), standard deviation, and interquartile range (IQR) and were compared using Student's t-test. Categorical variables are expressed as absolute number and percentages and analyzed using the $\chi^{2}$ test or the Fisher test as appropriate. All tests were two-sided, and a p-value of $<0.05$ was considered significant. Inter-reader reliability of the CT signs was evaluated using kappa-statistics.

To identify which radiological CT signs as covariates are most closely associated with AHF as the response variable, we employed the least absolute shrinkage and selection operation (LASSO) regression [25, 26]. LASSO is well suited to situations in which there is a high risk of collinearity, and it identifies the most robust predictor variables for the response variable by shrinking the regression coefficients toward zero. Only predictor variables with non-zero coefficients are retained in the final model. To test the robustness of the selected $\mathrm{CT}$ variables we performed LASSO in 60 different models using cross-validation. $\mathrm{CT}$ signs that came out in more than half of the 60 models were considered robust AHF markers (Suppl. Table 1).

We made an internal validation to report the sensitivity and specificity for detecting AHF based on zero or more significant $\mathrm{CT}$ signs identified from the LASSO model's most frequent parameters. These variables also entered a multiple logistic regression model with $\mathrm{AHF}$ as the response variable, and from these finally reported beta-coefficients we constructed a prediction score on a continuous scale and made an internal validation by using receiver-operating characteristic curves.

\section{Results}

\section{Population characteristics}

We screened 661 eligible patients with acute dyspnea. In total, 399 patients were excluded, including 272 patients due to unwillingness or inability to give written consent. In total, 232 were included with a complete dataset (Fig. 1) and a total of 102 (44\%) were assigned with AHF. Of the 102 patients with AHF, 30 had concomitant acute pulmonary disease ( 25 with lower respiratory tract infection, 3 with chronic obstructive pulmonary disease in exacerbation, 1 with cancer, and 1 with pulmonary fibrosis).

Patients with AHF were significantly older, had more cardiovascular diseases, and more clinical signs of AHF (Table 1). Many patients without AHF also had common clinical HF signs such as bilateral rales on auscultation, orthopnea, and cardiovascular disease. There was a significant difference in administered intravenous loop diuretics between AHF and non-AHF (Table 1).

\section{CT findings}

All 232 CT-examinations had adequate quality, although some artefacts were observed due to insufficient inspiration (9.5\%), motion (19.0\%), metal $(0.8 \%)$, and positioning $(1.7 \%)$. The mean radiation dose was $1.3 \mathrm{mSv}$, the median time to CT was $150 \mathrm{~min}$ (82.8-363.6), and the median time between $\mathrm{CT}$ and echocardiography was $110 \mathrm{~min}$ (IQR: 67.0-170.0).

\section{CT-score}

The univariate analyses revealed that nine of the evaluated CT signs were associated with AHF (Table 2). However, in repetitive LASSO regression models, just five of the CT-signs were repeatedly associated with AHF: bilateral groundglass opacification (odds ratio [OR] 2.07, 95\% CI 0.95-4.52), bilateral interlobular thickening (OR 
Table 1. Patient characteristics for the study population according to acute heart failure (AHF) versus no AHF.

\begin{tabular}{|c|c|c|c|}
\hline & No AHF $(n=130)$ & AHF (n = 102) & P overall \\
\hline Age [years] & $71.9 \pm 9.62$ & $77.8 \pm 9.58$ & $<0.001$ \\
\hline Sex (male) & $68(52.3 \%)$ & $63(61.8 \%)$ & 0.191 \\
\hline $\mathrm{SBP}[\mathrm{mmHg}]$ & $139 \pm 23.9$ & $150 \pm 31.4$ & 0.003 \\
\hline $\mathrm{DBP}[\mathrm{mmHg}]$ & $74.7 \pm 13.1$ & $80.5 \pm 16.7$ & 0.005 \\
\hline History of chronic heart failure & $16(12.3 \%)$ & $43(42.2 \%)$ & $<0.001$ \\
\hline Ischemic heart disease & $26(20.0 \%)$ & $33(32.4 \%)$ & 0.046 \\
\hline Atrial fibrillation & $25(19.2 \%)$ & $53(52.0 \%)$ & $<0.001$ \\
\hline COPD & $87(66.9 \%)$ & $33(32.4 \%)$ & $<0.001$ \\
\hline Hypertension & $72(55.4 \%)$ & $72(70.6 \%)$ & 0.026 \\
\hline \multicolumn{4}{|l|}{ Clinical findings } \\
\hline Orthopnea & $49(37.7 \%)$ & $65(63.7 \%)$ & $<0.001$ \\
\hline Bilateral rales & $30(23.1 \%)$ & $58(56.9 \%)$ & $<0.001$ \\
\hline Cough & $105(80.8 \%)$ & $69(67.6 \%)$ & 0.032 \\
\hline Fever & $21(16.2 \%)$ & $7(6.86 \%)$ & 0.051 \\
\hline Jugular vein distension & $1(0.77 \%)$ & $8(7.84 \%)$ & 0.012 \\
\hline Bilateral pedal edema & $24(18.5 \%)$ & $46(45.1 \%)$ & $<0.001$ \\
\hline \multicolumn{4}{|l|}{ Blood samples } \\
\hline Troponin-T [ng/L] (reference < 14 ng/L) & $17.0[11.5 ; 29.5]$ & $39.0[25.2 ; 60.8]$ & $<0.001$ \\
\hline C-reactive protein [mg/L] (reference $<10$ mg/L) & $23.9[6.00 ; 79.5]$ & $14.0[6.12 ; 47.9]$ & 0.094 \\
\hline Pro-natriuretic peptide [pmol/L] (reference < 100 pmol/L) & $41.1[16.2 ; 103]$ & $380[218 ; 790]$ & $<0.001$ \\
\hline \multicolumn{4}{|l|}{ Echocardiography } \\
\hline LVEF & $55.7 \pm 8.49$ & $42.5 \pm 16.2$ & $<0.001$ \\
\hline Tricuspid velocity & $248 \pm 74.7$ & $312 \pm 51.2$ & $<0.001$ \\
\hline E/é & $8.70[7.20 ; 11.0]$ & $15.0[11.1 ; 21.1]$ & $<0.001$ \\
\hline Indexed left atrial volume & $25.8 \pm 9.93$ & $46.3 \pm 12.6$ & $<0.001$ \\
\hline \multicolumn{4}{|l|}{ Cardiac dysfunction:* } \\
\hline Severe valve disease & $1(0.77 \%)$ & $8(7.84 \%)$ & 0.012 \\
\hline LVEF $<40$ & $5(3.85 \%)$ & $37(36.3 \%)$ & $<0.001$ \\
\hline LVEF 40-49 & $12(9.23 \%)$ & $13(12.7 \%)$ & 0.520 \\
\hline LVEF $\geq 50$ and LVH or LAH enlargement & $2(1.54 \%)$ & $44(43.1 \%)$ & $<0.001$ \\
\hline \multicolumn{4}{|l|}{ Intravenous loop diuretics } \\
\hline Before the low-dose CT & $5(4 \%)$ & $29(28 \%)$ & 0.007 \\
\hline Between the low-dose CT and echocardiography & $2(2 \%)$ & $7(7 \%)$ & 0.525 \\
\hline After the low-dose CT & $10(8 \%)$ & $39(38 \%)$ & 0.086 \\
\hline Did not receive at any time & $113(87 \%)$ & $27(26 \%)$ & 0.772 \\
\hline
\end{tabular}

Data are shown as mean \pm standard deviation or number (percentage) or median [interquartile range]. ${ }^{*} \mid \mathrm{t}$ was possible for a patient to have cardiac dysfunction, but without acute heart failure, if there were no clinical signs of heart failure or elevated left ventricular filling pressure; COPD - chronic obstructive pulmonary disease; CT — computed tomography; DBP — diastolic blood pressure; LAH — left atrial hypertrophy; LVH - left ventricular hypertrophy; LVEF — left ventricular ejection fraction; SBP - systolic blood pressure

11.67, 95\% CI 1.78-230.99), bilateral pleural effusion (OR 6.39, 95\% CI 1.98-22.85), enlarged heart (OR 20.38, 95\% CI 6.86-76.16), and increased vascular diameter (OR 4.49, 95\% CI 1.08-33.92) (Fig. 2, Suppl. Fig. 1A-D).

When applying the observer-independent technical AHF diagnosis as outcome (73 patients), the LASSO model selected four of the same distinct CT signs, but not bilateral ground glass opacities.

The reproducibility of the five CT signs were examined in terms of agreement between the two radiologists. The kappa value of the five $\mathrm{CT}$ signs were as follows: an enlarged heart 0.76 , bilateral interlobular thickening 0.64 , bilateral pleural ef- 


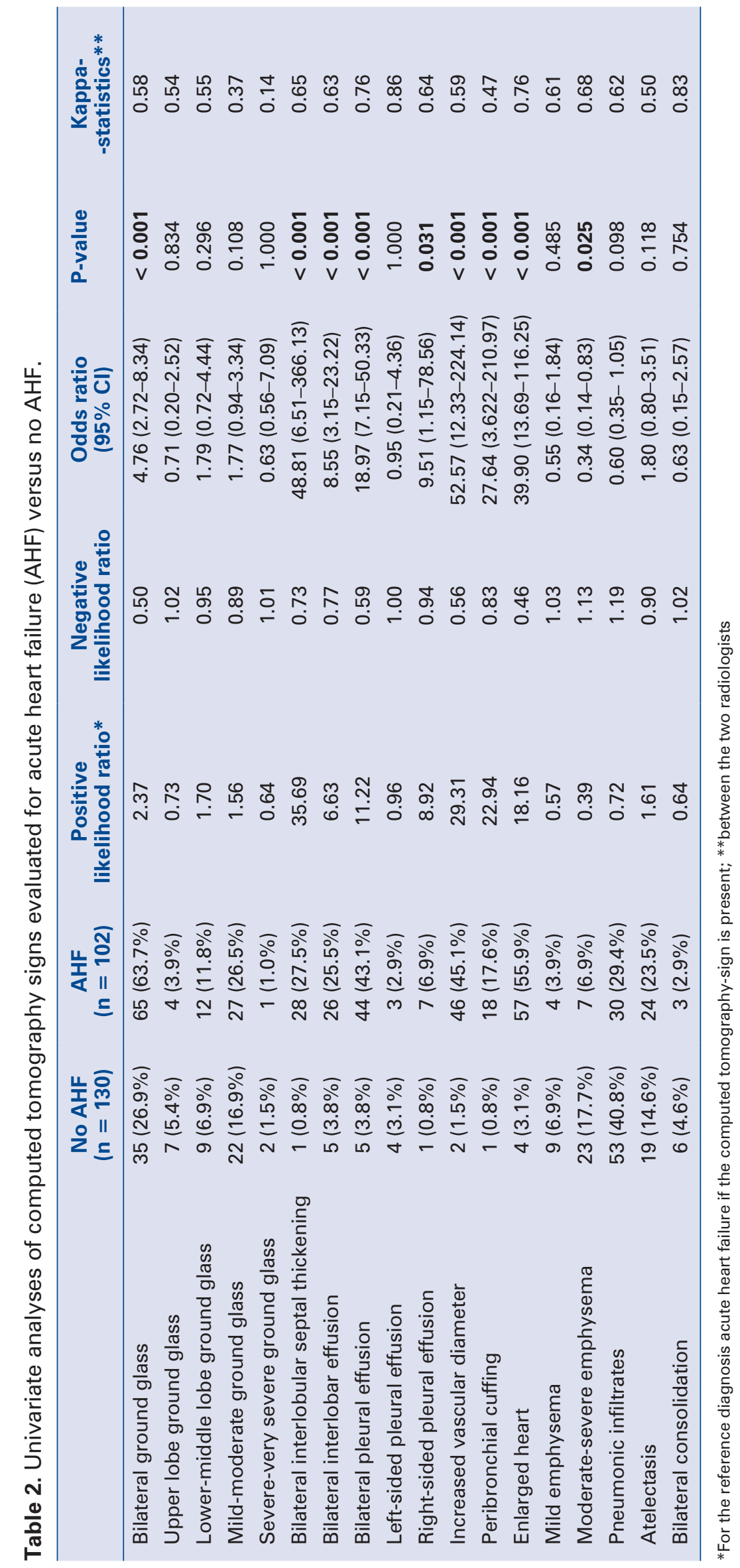




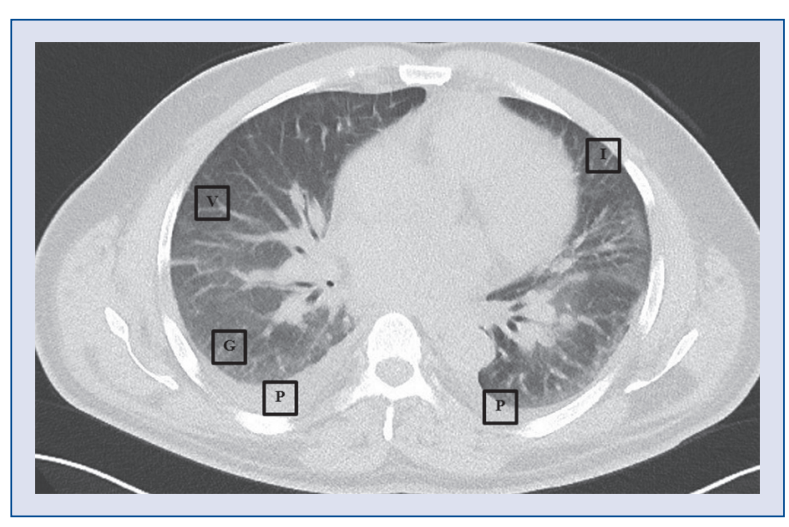

Figure 2. Four out of five essential computed tomography (CT) signs associated with acute heart failure illustrated in a patient admitted with acute dyspnea. The five CT signs were as follows: bilateral interlobular thickening (I), bilateral pleural effusion (P), increased vascular diameter (V), bilateral ground glass opacification (G), and an enlarged heart (sign not illustrated).

fusion 0.76 , increased vascular diameter 0.60 , and bilateral ground glass opacification 0.46 , respectively (Fig. 2, Suppl. Fig. 1A-D).

The five CT-signs were investigated in a multivariable logistic regression model using the reference $\mathrm{AHF}$ diagnosis as the response variable. Bilateral ground glass opacification had a univariate OR of 4.76 (2.72-8.34) but became nonsignificant in multivariable analysis (Fig. 3). The total sum of the beta-coefficients from the five CT signs ranged from 0 to 9.6, and the area under curve of this sum to diagnose AHF was 0.88 (Fig. 3).

Of all 232 included patients, a total of $88(38 \%)$ with adjudicated AHF had at least one of the five CT-signs, corresponding to a sensitivity and specificity of $86 \%(0.78-0.92)$ and $68 \%(0.60-0.76)$ for AHF (Table 3). Similarly, of all included patients, $68(29 \%)$ had adjudicated AHF and at least two of the five signs, corresponding to a sensitivity and specificity of $67 \%(0.57-0.76)$ and $97 \%(0.92-0.99)$, respectively (Table 3 ).

\section{Discussion}

Even though chest CT is increasingly used, there is currently no systematic approach for estimating AHF in dyspneic patients [16, 17]. Although many signs have previously been proposed, we report five essential CT signs: an enlarged heart, bilateral interlobular thickening, bilateral pleural effusion, increased intravascular diameter, and bilateral ground-glass. The current data suggest

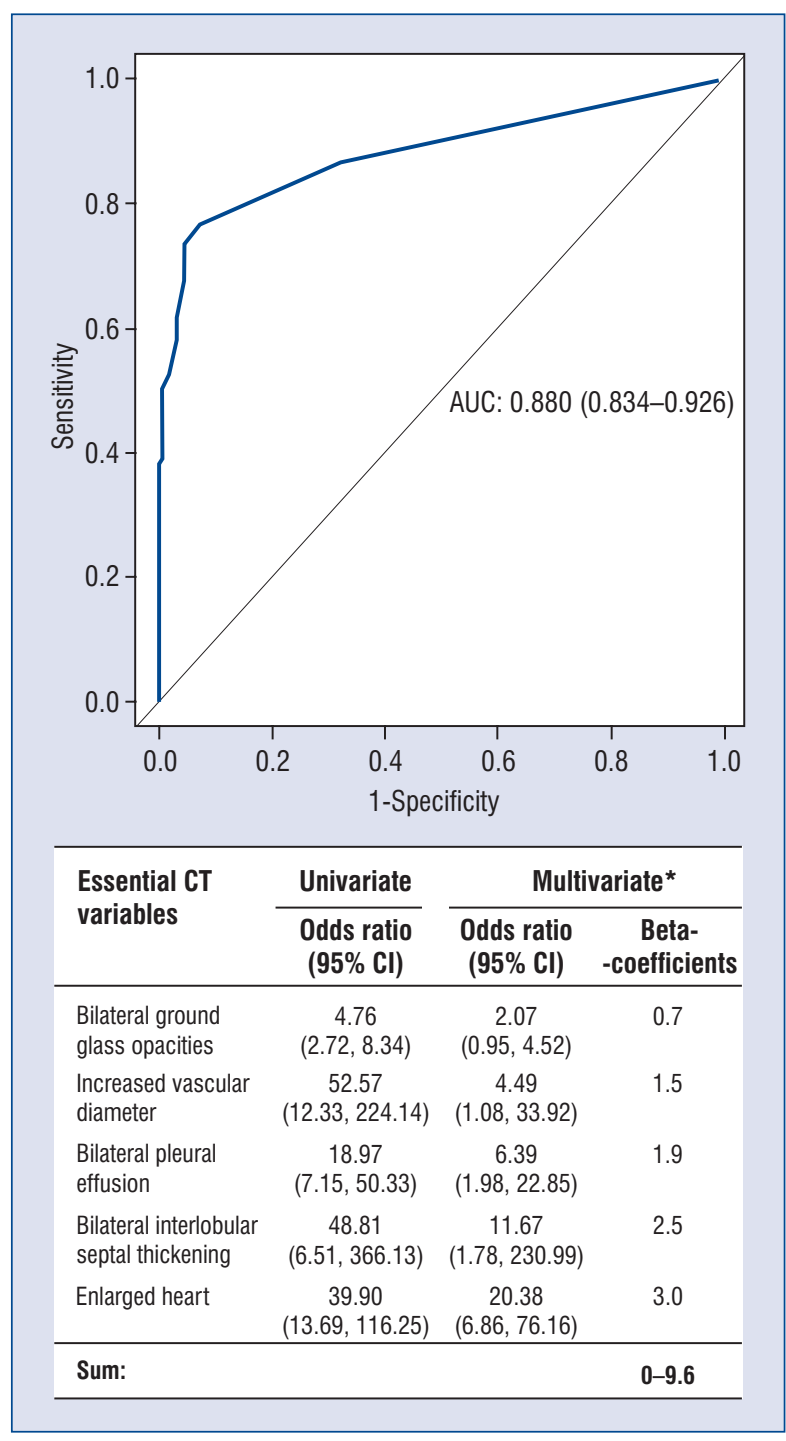

Figure 3. Receiver operating characteristics curve of the proposed computed tomography (CT) score to diagnose acute heart failure. The essential CT signs were weighted after their beta-coefficients in a logistic regression model, and the total sum ranged from 0 to 9.6; AUC - area under curve; $\mathrm{Cl}$ - confidence interval; ${ }^{*}$ the five CT variables.

that the absence of these signs indicates a low probability, one sign makes AHF highly probable, and two or more CT signs means almost certain AHF.

\section{Using CT to diagnose AHF}

Lung ultrasound is a quick bedside examination in which $B$ lines and bilateral pleural effusion have a high specificity for AHF [5]. Although the CXR and lung ultrasound are easily available in the acute setting, they are often inadequate for the evaluation of mild degrees of congestion [6, 27]. In 2014, CT was suggested as a reference method 
Table 3. The diagnostic accuracy of one or more of the five essential computed tomography (CT) signs.

\begin{tabular}{|c|c|c|c|c|c|c|c|c|}
\hline & \multicolumn{2}{|c|}{ AHF } & \multirow{2}{*}{$\begin{array}{c}\text { Sensitivity } \\
\text { (95\% Cl) }\end{array}$} & \multirow{2}{*}{$\begin{array}{c}\text { Specificity } \\
\text { (95\% CI) }\end{array}$} & \multirow{2}{*}{$\begin{array}{c}\text { PPV } \\
(95 \% \mathrm{Cl})\end{array}$} & \multirow{2}{*}{$\begin{array}{c}\text { NPV } \\
(95 \% \mathrm{Cl})\end{array}$} & \multirow{2}{*}{$\begin{array}{c}\text { PLR } \\
(95 \% \mathrm{Cl})\end{array}$} & \multirow{2}{*}{$\begin{array}{c}\text { NLR } \\
(95 \% \mathrm{CI})\end{array}$} \\
\hline & Yes & No & & & & & & \\
\hline \multicolumn{9}{|c|}{$\geq 1$ CT-signs } \\
\hline Yes & 88 & 41 & 0.86 & 0.68 & 0.68 & 0.86 & 2.74 & 0.20 \\
\hline No & 14 & 89 & $(0.78-0.92)$ & $(0.60-0.76)$ & $(0.59-0.76)$ & $(0.78-0.92)$ & $(2.10-3.56)$ & $(0.12-0.33)$ \\
\hline \multicolumn{9}{|c|}{$\geq 2$ CT-signs } \\
\hline Yes & 68 & 4 & 0.67 & 0.97 & 0.94 & 0.79 & 21.67 & 0.34 \\
\hline No & 34 & 126 & $(0.57-0.76)$ & $(0.92-0.99)$ & $(0.86-0.98)$ & $(0.72-0.85)$ & (8.18-57.41) & $(0.26-0.45)$ \\
\hline
\end{tabular}

AHF — acute heart failure; $\mathrm{Cl}$ - confidence interval; PPV — positive predictive value; NPV - negative predictive value; PLR - positive likelihood ratio; NLR — negative likelihood ratio

for evaluating undifferentiated dyspnea [4], and it may be useful as a secondary examination. However, there is no recommended scoring system, published systematic review, or metanalysis on the subject, no systematic approach to support or reject $\mathrm{AHF}$ by $\mathrm{CT}$ in breathless patients, and no guide on how to weigh the importance of each sign. Several selected radiological signs have previously been described as markers of HF: cardiomegaly [15], ground glass opacification [11, 15, 28], thickening of intra- and interlobular septa $[11,13,15$, 28], mosaic pattern of attenuation [11], peribronchial cuffing [11, 13, 28], pleural effusion [13, 15], consolidations [11, 15], and increased peripheral vascular diameter [11, 13, 15, 28]. Nonetheless, these previous studies used older generation CT scanners and were often more than a decade old, retrospective-, case-studies, or only included patients with already known cardiac disease [11-15].

\section{Validity of CT signs}

We performed a prospective study, including a broad spectrum of dyspnoeic patients. Consecutive sampling was important because some CTsigns, such as ground glass opacification, pleural effusion, and consolidations, are non-specific and may be seen in both AHF and pneumonia (Table 2) $[22,29]$. Notably, bilateral ground glass opacity was a mediocre sign of AHF and not significant on multivariable analysis. Still, the absence of bilateral ground glass opacification and the four other signs indicates a low probability of AHF.

We confirm the association between cardiomegaly and AHF, but cardiomegaly was not a particularly strong marker. However, if all five markers are evaluated, it is possible to discard or raise suspicion of AHF. Hence, for the first time, we report how these signs should be prioritized when evaluating a CT for $\mathrm{HF}$.
The agreement and kappa values of the five CT signs for AHF ranged from moderate to substantial. Hence, determining the probability of AHF from $\mathrm{CT}$ requires all the signs to be examined. It is a novel observation, and a simplification, that all diagnostic information for AHF is contained in just five CT signs.

Although not externally validated, the CT-signs were identified by cross-validation and random sampling. Furthermore, a sensitivity analyses based on a technical observer-independent AHF diagnosis indicated a high stability of the CT signs.

\section{The reference diagnosis of $\mathrm{AHF}$}

Previous studies compared CT signs against various aspects of congestive $\mathrm{HF}$ either by retrospective reviews [11] or in selected patients. Some studies examined patients with very specific criteria of HF (pulmonary wedge pressure or chronic $\mathrm{HF}$ ) or more vague criteria (LVEF $<30 \%$, improvement of pulmonary congestion on CXR, elevated central pressure, physical examination) [30]. Some studies may have been biased by using only medical record review [15], clinical history, and the resolution of radiologic abnormalities after diuretics [28] or retrospective analysis of patients with clinical profiles of pulmonary edema [11]. All studies carry a high risk of bias because the spectrum of CT signs could have indirectly affected the decision to initiate treatment and thus the reference diagnosis.

We acknowledge the difficulty in assessing a diagnosis of AHF without direct or indirect bias from radiology. We did our best to apply a stateof-the-art diagnosis [1, 3] with a strong emphasis on the comprehensive echocardiogram. Still, 25 (11\%) patients had indeterminate LV filling pressures and were evaluated by a cardiologist to adjudicate the diagnosis by reviewing the medical 
record including pro-BNP and response to treatment. Importantly, this adjudication was done independently of radiology findings including the CXR because it is highly correlated with chest CT. Furthermore, we included an analysis based on an observer-independent technical AHF diagnosis, which corroborates our findings.

\section{Study strengths and limitations}

The first strength is that this is the first study to systematically and prospectively examine CT for objective evidence of AHF in consecutive dyspneic patients. Second, we used an CT in every patient without a previous selection related to the presumed cause of breathlessness. Third, the median time between CT and echocardiography was only $110 \mathrm{~min}$. Such measures are difficult to attain in an acute clinical setting but necessary to ensure that patients had a similar cardiovascular state. Fourth, the CT images were reviewed by two independent thorax radiologists from two different hospitals.

Nonetheless, the study also had some limitations. First, the study was a single-center study, with daytime sampling. The majority of patients were excluded due to unwillingness or inability to give informed consent (e.g., dementia). The low inclusion rate is a common problem in acute studies where patients have little time to understand and provide full informed consent. Another reason for the low inclusion rate was exclusion of patients with acute coronary syndrome [31]. The results therefore relate to intermediate ill acute patients who often pose a considerable diagnostic challenge. Second, patients with cor-pulmonale or right-sided HF were not excluded if they fulfilled the AHF diagnosis, although such patients are not expected to have pulmonary congestion. Therefore, some of these patients were classified as negative by the CT, which partly explains why AHF cannot be completely ruled out with the CT (negative likelihood ratio: 0.2) (Table 3). Third, the significant CT signs were identified by crossvalidation and random sampling procedures, which corroborate robustness. However, the reported beta-coefficients may be optimistic because they are fitted to the present population.

\section{Conclusions}

This study provides a standardized approach to ascertain objective evidence of AHF on CT in patients with acute dyspnea. Only five essential CT signs are needed to evaluate the likelihood AHF.
The absence of these signs indicates a low probability, one sign makes AHF highly probable, and two or more CT-signs means almost certain AHF.

\section{Acknowledgments}

We would like to thank all the involved departments as well as the hospital staff for supporting this project.

\section{Funding}

This project was partially supported by grants from the Bispebjerg \& Frederiksberg Research Funds, "Helsefonden" and "Snedkermester Sophus Jacobsen \& hustru Astrid Jacobsens Fond”, Copenhagen. Sponsors did not play a role in the design or conduct of the study, nor in the collection, interpretation, analysis, or review of data, and they did not approve the manuscript for publication.

Conflict of interest: Olav Wendelboe Nielsen has received fees for speaking and advisory board membership by AstraZeneca and Novartis not related to the current study. Christian Torp-Pedersen has received CTP grants from Buyers and NovoNordisk not related to the current study. All other authors have no conflict of interest to declare.

\section{References}

1. Bozkurt B, Coats AJ, Tsutsui H, et al. Universal Definition and Classification of Heart Failure: A Report of the Heart Failure Society of America, Heart Failure Association of the European Society of Cardiology, Japanese Heart Failure Society and Writing Committee of the Universal Definition. J Card Fail. 2021: S1071-S9164, doi: 10.1016/j.cardfail.2021.01.022, indexed in Pubmed: 33663906.

2. McDonagh T, Metra M, Adamo M. The 'Ten Commandments' of the 2021 ESC Guidelines for the diagnosis and treatment of acute and chronic heart failure. Eur Heart J. 2021, doi: 10.1093/ eurheartj/ehab853, indexed in Pubmed: 34447992.

3. Ponikowski P, Voors AA, Anker SD, et al. 2016 ESC Guidelines for the diagnosis and treatment of acute and chronic heart failure: The Task Force for the diagnosis and treatment of acute and chronic heart failure of the European Society of Cardiology (ESC). Developed with the special contribution of the Heart Failure Association (HFA) of the ESC. Eur J Heart Fail. 2016; 18(8): 891-975, doi: 10.1002/ejhf.592, indexed in Pubmed: 27207191.

4. Cardinale L, Priola AM, Moretti F, et al. Effectiveness of chest radiography, lung ultrasound and thoracic computed tomography in the diagnosis of congestive heart failure. World J Radiol. 2014; 6(6): 230-237, doi: 10.4329/wjr.v6.i6.230, indexed in Pubmed: 24976926.

5. Miger KC, Fabricius-Bjerre A, Maschmann CP, et al. Clinical applicability of lung ultrasound methods in the emergency department to detect pulmonary congestion on computed tomography. Ultraschall Med. 2021; 42(3): e21-e30, doi: 10.1055/a1021-1470, indexed in Pubmed: 31648347. 
6. Mullens W, Damman K, Harjola VP, et al. The use of diuretics in heart failure with congestion - a position statement from the Heart Failure Association of the European Society of Cardiology. Eur J Heart Fail. 2019; 21(2): 137-155, doi: 10.1002/ejhf.1369, indexed in Pubmed: 30600580.

7. Salvatore M, Henschke CI, Yip R, et al. JOURNAL CLUB: evidence of interstitial lung disease on low-dose chest CT images: prevalence, patterns, and progression. Am J Roentgenol. 2016; 206(3): 487-494, doi: 10.2214/AJR.15.15537, indexed in Pubmed: 26700157.

8. Seidel J, Bissell MB, Vatturi S, et al. Retrospective analysis of emergency computed tomography imaging utilization at an academic centre: an analysis of clinical indications and outcomes. Can Assoc Radiol J. 2019; 70(1): 13-22, doi: 10.1016/j. carj.2018.10.004, indexed in Pubmed: 30691557.

9. Bellolio MF, Heien HC, Sangaralingham LR, et al. Increased computed tomography utilization in the emergency department and its association with hospital admission. West J Emerg Med. 2017; 18(5): 835-845, doi: 10.5811/westjem.2017.5.34152, indexed in Pubmed: 28874935.

10. Aziz W, Claridge S, Ntalas I, et al. Emerging role of cardiac computed tomography in heart failure. ESC Heart Fail. 2019; 6(5): 909-920, doi: 10.1002/ehf2.12479, indexed in Pubmed: 31400060.

11. Ribeiro CMC, Marchiori E, Rodrigues R, et al. Hydrostatic pulmonary edema: high-resolution computed tomography aspects. J Bras Pneumol. 2006; 32(6): 515-522, doi: 10.1590/s180637132006000600008, indexed in Pubmed: 17440672.

12. Storto ML, Kee ST, Golden JA, et al. Hydrostatic pulmonary edema: high-resolution CT findings. Am J Roentgenol. 1995; 165(4): 817-820, doi: 10.2214/ajr.165.4.7676973, indexed in Pubmed: 7676973.

13. Marano R, Pirro F, Silvestri V, et al. Comprehensive CT cardiothoracic imaging: a new challenge for chest imaging. Chest. 2015; 147(2): 538-551, doi: 10.1378/chest.14-1403, indexed in Pubmed: 25644907.

14. Clark TJ, Suby-Long T. Computed tomography of acute heart failure: A novel case and literature review. Radiol Case Rep. 2015; 10(1): 1065, doi: 10.2484/rcr.v10i1.1065, indexed in Pubmed: 27408665.

15. Lewin S, Goldberg L, Dec GW. The spectrum of pulmonary abnormalities on computed chest tomographic imaging in patients with advanced heart failure. Am J Cardiol. 2000; 86(1): 98-100, doi: 10.1016/s0002-9149(00)00837-7, indexed in Pubmed: 10867103.

16. Jokerst C, Chung JH, Ackman JB, et al. Expert Panel on Thoracic Imaging:. ACR Appropriateness Criteria Acute Respiratory Illness in Immunocompetent Patients. J Am Coll Radiol. 2018; 15(11S): S240-S251, doi: 10.1016/j.jacr.2018.09.012, indexed in Pubmed: 30392593.

17. Dr Joachim Feger and Dr Yuranga Weerakkody. Radiopaedia: Congestive cardiac failure. https://radiopaedia.org/articles/congestive-cardiac-failure (cited 2021 Mar 10).
18. Hendee WR, O'Connor MK. Radiation risks of medical imaging: separating fact from fantasy. Radiology. 2012; 264(2): 312-321, doi: 10.1148/radiol.12112678, indexed in Pubmed: 22821690.

19. Sundhedsstyrelsen S. Stråling i sundhedsvidenskabelige fors $\varnothing$ g. Den Natl Vidensk Komité. 2011; 2(2-4): 2-4.

20. Nagueh SF, Smiseth OA, Appleton CP, et al. Recommendations for the Evaluation of Left Ventricular Diastolic Function by Echocardiography: An Update from the American Society of Echocardiography and the European Association of Cardiovascular Imaging. Eur Heart J Cardiovasc Imaging. 2016; 17(12): 1321-1360, doi: 10.1093/ehjci/jew082, indexed in Pubmed: 27422899.

21. Nagueh SF, Appleton CP, Gillebert TC, et al. Recommendations for the evaluation of left ventricular diastolic function by echocardiography. Eur J Echocardiogr. 2009; 10(2): 165-193, doi: 10.1093/ejechocard/jep007, indexed in Pubmed: 19270053.

22. Hansell DM, Bankier AA, MacMahon H, et al. Fleischner Society: glossary of terms for thoracic imaging. Radiology. 2008; 246(3): 697-722, doi: 10.1148/radiol.2462070712, indexed in Pubmed: 18195376.

23. Christe A, Charimo-Torrente J, Roychoudhury K, et al. Accuracy of low-dose computed tomography (CT) for detecting and characterizing the most common CT-patterns of pulmonary disease. Eur J Radiol. 2013; 82(3): e142-e150, doi: 10.1016/j. ejrad.2012.09.025, indexed in Pubmed: 23122673.

24. Team RCRF for SC. R: A Language and Environment for Statistical Computing [Internet]. 2019. https://www.r-project.org/.

25. Tibshirani R. Regression Shrinkage and Selection Via the Lasso. J R Stat Soc Ser B Methodological. 1996; 58(1): 267-288, doi: 10.1111/j.2517-6161.1996.tb02080.x.

26. Hastie T, Tibshirani R, James G, Witten D. An Introduction to Statistical Learning, Vol. 102. Springer Texts, New York 2006: 618.

27. Gheorghiade M, Follath F, Ponikowski P, et al. Assessing and grading congestion in acute heart failure: a scientific statement from the acute heart failure committee of the heart failure association of the European Society of Cardiology and endorsed by the European Society of Intensive Care Medicine. Eur J Heart Fail. 2010; 12(5): 423-433, doi: 10.1093/eurjhf/hfq045, indexed in Pubmed: 20354029.

28. Storto ML, Kee ST, Golden JA, et al. Hydrostatic pulmonary edema: high-resolution CT findings. Am J Roentgenol. 1995; 165(4): 817-820, doi: 10.2214/ajr.165.4.7676973, indexed in Pubmed: 7676973.

29. Jany B, Welte T. Pleural effusion in adults-etiology, diagnosis, and treatment. Dtsch Arztebl Int. 2019; 116(21): 377-386, doi: 10.3238/arztebl.2019.0377, indexed in Pubmed: 31315808.

30. Slanetz PJ, Truong M, Shepard JA, et al. Mediastinal lymphadenopathy and hazy mediastinal fat: new CT findings of congestive heart failure. Am J Roentgenol. 1998; 171(5): 1307-1309, doi: 10.2214/ajr.171.5.9798869, indexed in Pubmed: 9798869.

31. Steg PG, James SK, Atar D, et al. ESC Guidelines for the management of acute myocardial infarction in patients presenting with ST-segment elevation. Eur Heart J. 2012; 33(20): 2569-619, doi: 10.1093/eurhearti/ehs215, indexed in Pubmed: 22922416. 